\title{
Metabolomic signatures distinguish the impact of formula carbohydrates on disease outcome in a preterm piglet model of NEC
}

Lee Call', Barbara Stoll ${ }^{1}$, Berthe Oosterloo ${ }^{1}$, Nadim Ajami ${ }^{3}$, Fariha Sheikh², Anja Wittke ${ }^{4}$, Rosaline Waworuntu', Brian Berg ${ }^{4}$, Joseph Petrosino ${ }^{3}$, Oluyinka Olutoye ${ }^{2}$ and Douglas Burrin ${ }^{1 *}$ (D)

\begin{abstract}
Background: Major risk factors for necrotizing enterocolitis (NEC) include premature birth and formula feeding in the context of microbial colonization of the gastrointestinal tract. We previously showed that feeding formula composed of lactose vs. corn syrup solids protects against NEC in preterm pigs; however, the microbial and metabolic effects of these different carbohydrates used in infant formula has not been explored.

Objective: Our objective was to characterize the effects of lactose- and corn syrup solid-based formulas on the metabolic and microbial profiles of preterm piglets and to determine whether unique metabolomic or microbiome signatures correlate with severity or incidence of NEC.

Design/methods: Preterm piglets (103 days gestation) were given total parenteral nutrition (2 days) followed by gradual (5 days) advancement of enteral feeding of formulas matched in nutrient content but containing either lactose (LAC), corn syrup solids (CSS), or 1:1 mix (MIX). Gut contents and mucosal samples were collected and analyzed for microbial profiles by sequencing the V4 region of the $16 \mathrm{~S}$ rRNA gene. Metabolomic profiles of cecal contents and plasma were analyzed by LC/GC mass spectrometry.

Results: NEC incidence was 14, 50, and 44\% in the LAC, MIX, and CSS groups, respectively. The dominant classes of bacteria were Bacilli, Clostridia, and Gammaproteobacteria. The number of observed OTUs was lowest in colon contents of CSS-fed pigs. CSS-based formula was associated with higher Bacilli and lower Clostridium from clusters XIVa and XI in the colon. NEC was associated with decreased Gammaproteobacteria in the stomach and increased Clostridium sensu stricto in the ileum. Plasma from NEC piglets was enriched with metabolites of purine metabolism, aromatic amino acid metabolism, and bile acids. Markers of glycolysis, e.g., lactate, were increased in the cecal contents of CSs-fed pigs and in plasma of pigs which developed NEC.
\end{abstract}

Conclusions: Feeding formula containing lactose is not completely protective against NEC, yet selects for greater microbial richness associated with changes in Bacilli and Clostridium and lower NEC incidence. We conclude that feeding preterm piglets a corn syrup solid vs. lactose-based formula increases the incidence of NEC and produces distinct metabolomic signatures despite modest changes in microbiome profiles.

Keywords: Necrotizing enterocolitis, Infant formula, Lactose, Maltodextrin, Corn syrup solids, Clostridium, Lactate

\footnotetext{
* Correspondence: doug.burrin@ars.usda.gov

'USDA-ARS Children's Nutrition Research Center, Department Pediatric

Gastroenterology, Hepatology, and Nutrition, Baylor College of Medicine,

1100 Bates Ave, Houston, TX 77030, USA

Full list of author information is available at the end of the article
}

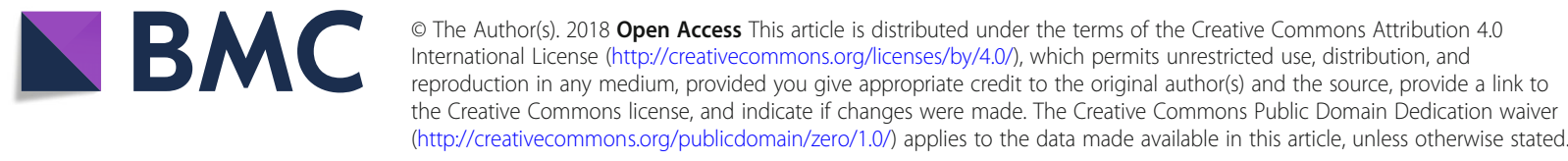




\section{Background}

Necrotizing enterocolitis (NEC) persists as a major gastrointestinal disease among preterm infants that is characterized by a severe inflammatory response leading to necrosis of intestinal tissue and major morbidity [1, 2]. At least two dominant factors that are thought to be causally associated with NEC are bacterial colonization and enteral formula feeding. Recent evidence suggests that the preterm infant gut is not sterile [3, 4], but clearly the process of birth and especially enteral feeding sets off a cascade of physiological processes that lead to increased numbers and diversity of microbes. In animal models, NEC-like disease does not occur in animals raised germ-free [5-7], or when given broad-spectrum antibiotics [8]. In healthy, term newborn infants, gut microbial colonization can facilitate normal development of the host immune system [9-11]. However, in preterm infants, gut microbes trigger an exaggerated proinflammatory response leading to necrosis and injury because of immature intestinal innate immune function.

Microbial colonization is a critical element in NEC pathogenesis, and this has spawned widespread investigation to find specific bacteria linked to NEC [12-24]. Several reports in the past 10 years have shown associations between gut microbes and NEC using molecular, culture-independent approaches based on 16S rRNA gene sequence or metagenomic analysis [13, 25-27]. Despite these studies, no single species or uniform microbial signature has been linked to NEC; however, some taxonomic groups have been associated including Proteobacteria [28], Gammaproteobacteria [29], and Clostridium and Staphylococcus sp. [24]. This question has proven difficult due to several factors including the low diversity yet high inter-individual variation characteristic of the neonatal microbiota [30-32], differences in analysis methods (i.e., culture- vs. non-culture-based, different sequencing platforms) $[33,34]$, and the challenges of interpreting results across the various animal models and human patients, each harboring its own co-evolved microbiota [35-38]. Many of these studies also suggest that differences exist in the functional metabolic capacity of microbial communities with similar $16 \mathrm{~S}$ rRNA gene signatures. More recently, studies have pursued a dual approach using non-targeted, metabolomic profiling of blood, urine, or stool coupled with genomic sequence analysis of fecal microbial community structure in infants with NEC [13, 25-27]. Some of these studies have shown concordance between select metabolites and $16 \mathrm{~S}$ microbiome signatures in NEC and healthy infants.

Another dominant factor in NEC pathogenesis is diet, and numerous studies show that breast milk significantly lowers the incidence of NEC in preterm infants, in comparison to milk-based enteral formulas [39-43]. The primary carbohydrates in breast milk are lactose and oligosaccharides, whereas preterm infant formulas contain only a mixture $(\sim 50 / 50 \%)$ of lactose and maltodextrin or corn syrup solids. Corn syrup solids and maltodextrin are short $(<20)$ chains of glucose that are considered easier to digest than lactose in preterm neonates who often have lower lactase expression compared to term infants [44-47]. Human milk oligosaccharides have received considerable attention in support of normal microbiome development and as a protective component of human milk against NEC [48, 49]. However, the specific effects of lactose and corn syrup solids on the developing infant gut microbiome are largely unknown. Importantly, we previously showed in a preterm piglet model that feeding formulas containing lactose reduced the incidence of NEC by threefold compared to those fed maltodextrin [50]. The aim of the current study was to extend these findings and use the dual approach of microbiome and non-targeted metabolomic profiling to interrogate the relationships between dietary carbohydrates, gut microbiome community structure, and NEC pathogenesis in a preterm, neonatal piglet model. The preterm piglet is a well-established model to study NEC because it incorporates the necessary elements of prematurity and gut microbial colonization and employs relevant clinical features of enteral and parenteral nutrition in a species with close homology in physiology, intestinal anatomy, and metabolism to human preterm infants $[6,8,51-54]$. These reports have shown that the clinical and histopathologic NEC phenotype in pigs is remarkably similar to NEC in human infants.

\section{Results}

\section{NEC incidence and severity highest in CSS-fed piglets}

The composition of the formulas fed in each dietary group is shown in Table 1. A total of 78 pigs were included in this experiment. The incidence of NEC within the LAC,

Table 1 Ingredient and macronutrient composition of formulas containing lactose (LAC), corn syrup solids (CSS), or mixture (MIX)

\begin{tabular}{llll}
\hline & LAC & MIX & CSS \\
\hline Lactose & 60.0 & 30.0 & - \\
Corn syrup solids & - & 30.0 & 60.0 \\
MCT oil & 24.7 & 24.7 & 24.7 \\
Soybean oil & 18.2 & 18.2 & 18.2 \\
High oleic (sunflower) oil & 16.1 & 16.1 & 16.1 \\
Casein protein hydrolysate & 28.7 & 28.7 & 28.7 \\
Whey protein isolate & 37.3 & 37.3 & 37.3 \\
Protein & 66.0 & 66.0 & 66.0 \\
Fat & 59.0 & 59.0 & 59.0 \\
Carbohydrate & 60.0 & 60.0 & 60.0 \\
\hline
\end{tabular}

Components are listed as $\mathrm{g} / \mathrm{L}$. All additional macrominerals, trace minerals, and vitamins are formulated to meet or exceed the requirements of neonatal piglets 
MIX, and CSS groups were 14\% (3/21), 50\% (6/12), and $44 \%$ (20/45), respectively (Fig. 1a). By analyzing the rates of fatal cases of NEC within each group, we found that the Kaplan-Meier survival curves of the LAC and CSS groups differ by the log-rank test $(p=0.027)$ (Fig. 1b). This also was reflected in the earlier time of death or euthanasia due to NEC in CSS and MIX pigs than LAC pigs (Table 2). The jejunum, ileum, and colon mean gross severity scores as determined at the time of death or euthanasia were lower in the LAC group compared to the CSS group; the LAC group's ileum scores were also lower compared to the MIX group, while no difference was detected for the stomach (Fig. 1c). The severity of NEC lesions were subsequently confirmed by histological scoring using an index previously adapted for this model (Fig. 1c) [55].

\section{Morphology, histology, and inflammatory gene expression} Within the CSS-fed piglets, those animals that subsequently developed NEC had an average lower body weight at birth (926 vs. $1066 \mathrm{~g}$ ) and at euthanasia for NEC or end of study (1203 vs. 1530 g) compared to those that did not develop NEC (Table 2). There were no differences in birth weight, end of study weights, or rate of weight gain among healthy pigs in any of the formula groups. In gut morphometry, the most pronounced effect of NEC occurred in the terminal ileum villus height and crypt depth, both measures being lower in the piglets with NEC compared to healthy, regardless of which formula they received. It is important to note that although villus height does tend to increase with feedings after birth, and indeed terminal ileum measurements for all healthy pigs at the end of study are increased compared to the average at birth, $411 \pm 33 \mu \mathrm{m}$ (data not shown), the effect of NEC is to decrease villus height regardless of diet or time of death (Additional file 1). Lower measures of jejunum villus height were also observed in NEC vs. healthy piglets. We observed decreased jejunum crypt depths for NEC vs. healthy within the MIX- and CSS-fed piglets. Colonic crypt depths of NEC piglets were greatest in the LAC group vs. MIX and CSS and somewhat surprisingly were also greater compared to the healthy LAC-fed piglets.

The expression of a set of inflammatory-related genes was not significantly different across the various groups in this study. However, there was a trend for greater expression of IL-1b, IL-6, and IL-8 in samples

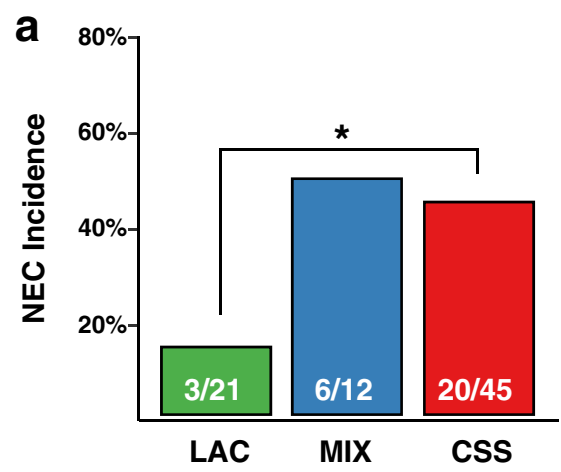

C

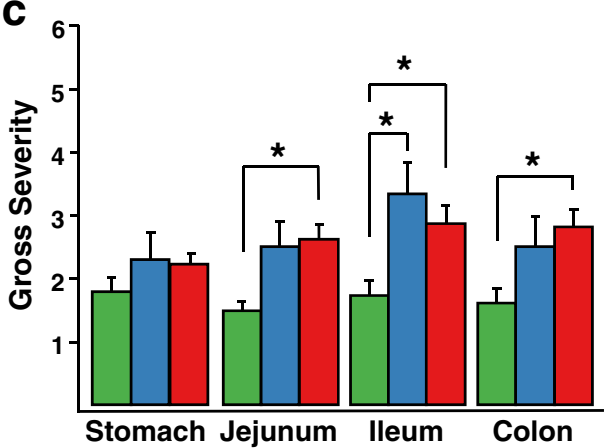

b
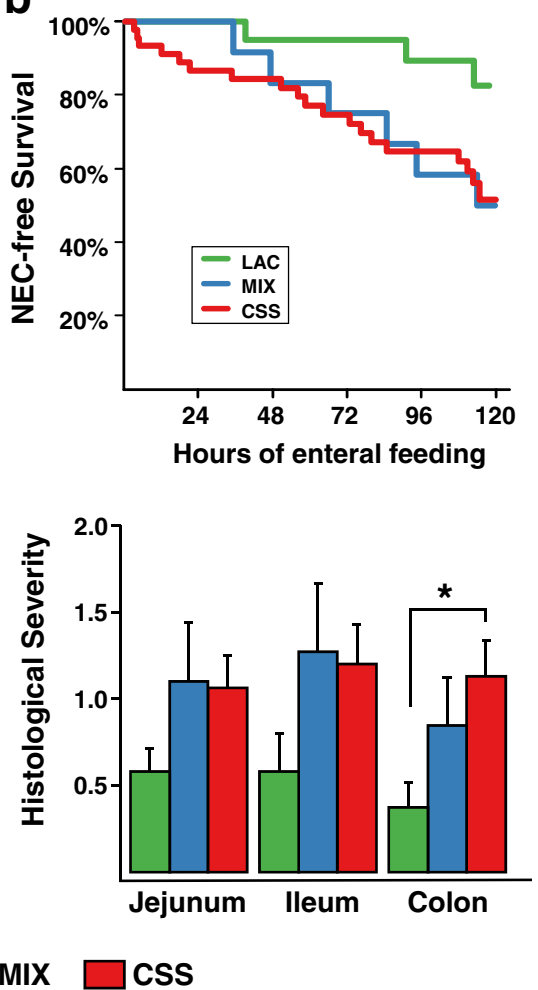

Fig. 1 Phenotypic outcomes of the study. a NEC incidence within each of the three formula groups; Fisher's exact test. b Kaplan-Meir analysis comparing survival time before euthanizing for NEC across the three formula groups; log-rank test. c Gross and histological NEC severity scores across GI regions for the three formula groups. For the gross severity, each of the Gl regions is assessed at the time of dissection and assigned a score of 1-2 (healthy tissue), 3-4 (moderate inflammation), or 5-6 (pneumatosis and necrosis). For histological severity, $\mathrm{H} \&$ E-stained tissue sections are scored as 0 (no damage), or from a range of 1 to 4 based on extent of necrosis, villus blunting, and pneumatosis. Values are mean +/- standard error of the mean; linear model with birthweight and farm as covariates and Tukey's post hoc comparisons 
Table 2 Body weights and gut morphometry of piglets fed formulas containing lactose (LAC), corn syrup solids (CSS), or mixture (MIX)

\begin{tabular}{|c|c|c|c|}
\hline & LAC & MIX & CSS \\
\hline \multicolumn{4}{|c|}{ Number pigs/group } \\
\hline Healthy & 18 & 6 & 25 \\
\hline NEC & 3 & 6 & 20 \\
\hline \multicolumn{4}{|c|}{ Time of death, hr post-feeding } \\
\hline Healthy & $106 \pm 7$ & $121 \pm 1$ & $104 \pm 6$ \\
\hline NEC & $83 \pm 23$ & $75 \pm 12 \dagger$ & $62 \pm 9+$ \\
\hline \multicolumn{4}{|c|}{ Birth weight (g) } \\
\hline Healthy & $934 \pm 45$ & $1011 \pm 62$ & $1066 \pm 45$ \\
\hline NEC & $872 \pm 28$ & $1010 \pm 108$ & $926 \pm 50 \dagger$ \\
\hline \multicolumn{4}{|c|}{ Final weight (g) } \\
\hline Healthy & $1382 \pm 81$ & $1511 \pm 86$ & $1530 \pm 79$ \\
\hline NEC & $1203 \pm 116$ & $1310 \pm 122$ & $1203 \pm 97 \dagger$ \\
\hline \multicolumn{4}{|c|}{ Weight gain $\left(\mathrm{g} \cdot \mathrm{kg}^{-1} \cdot\right.$ day $\left.^{-1}\right)$} \\
\hline Healthy & $104 \pm 6$ & $99 \pm 6$ & $94 \pm 6$ \\
\hline NEC & $106 \pm 17$ & $86 \pm 30$ & $61 \pm 20$ \\
\hline \multicolumn{4}{|c|}{ Proximal jejunum } \\
\hline \multicolumn{4}{|c|}{ Villus height $(\mu \mathrm{m})$} \\
\hline Healthy & $467 \pm 34$ & $427 \pm 39$ & $569 \pm 33$ \\
\hline NEC & $314 \pm 25+$ & $305 \pm 32 \dagger$ & $330 \pm 38 t$ \\
\hline \multicolumn{4}{|c|}{ Crypt depth $(\mu \mathrm{m})$} \\
\hline Healthy & $94 \pm 3$ & $107 \pm 8$ & $102 \pm 5$ \\
\hline NEC & $75 \pm 8$ & $79 \pm 9+$ & $83 \pm 4 \dagger$ \\
\hline \multicolumn{4}{|c|}{ Terminal ileum } \\
\hline \multicolumn{4}{|c|}{ Villus height ( $\mu \mathrm{m})$} \\
\hline Healthy & $537 \pm 21$ & $518 \pm 12$ & $634 \pm 45$ \\
\hline NEC & $179 \pm 52+$ & $277 \pm 55 \dagger$ & $291 \pm 46 \dagger$ \\
\hline \multicolumn{4}{|c|}{ Crypt depth $(\mu \mathrm{m})$} \\
\hline Healthy & $114 \pm 6$ & $131 \pm 12$ & $121 \pm 6$ \\
\hline NEC & $97 \pm 3+$ & $75 \pm 9+$ & $82 \pm 6+$ \\
\hline \multicolumn{4}{|l|}{ Colon } \\
\hline \multicolumn{4}{|c|}{ Crypt depth $(\mu \mathrm{m})$} \\
\hline Healthy & $232 \pm 14$ & $183 \pm 16$ & $216 \pm 9$ \\
\hline NEC & $314 \pm 2^{a} \dagger$ & $148 \pm 5^{b}$ & $213 \pm 14^{c}$ \\
\hline
\end{tabular}

Values are mean \pm standard error of the mean. Superscripts $a, b$, and c designate the results of Tukey's post hoc test comparing across groups; groups sharing letters are not significantly different (no superscripts if no differences were detected for that row). The cross, + , identifies differences between Healthy and NEC within a group, by student's $t$ test. An alpha level of 0.05 was used for all tests

of distal ileum tissue from NEC piglets fed CSS formula (Additional file 2).

\section{Impact of formula carbohydrates on preterm piglet microbiome}

We collected samples from along the gastrointestinal tract from a subset of the pigs treated in order to assess the development of microbial communities in association with feeding these formulas. We observed the highest number of OTUs in the colons of the healthy LAC- and MIX-fed pigs, median 85 and 92 OTUs, respectively, compared to 51 in CSS-fed pigs (Fig. 2). We did not observe any differences in alpha diversity due to feeding different formulas (data not shown).

Overall, most of the samples from both luminal contents as well as tissue mucosa were dominated by two classes of bacteria--Gammaproteobacteria and Clostridia, except for samples of stomach contents, where Bacilli make up a significant portion of the community. The relative abundance of Bacilli was significantly higher in the stomach (median 44\%) than in either the ileum (7\%) or colon contents (5\%). In addition, the relative abundance of Bacilli in colon contents and tissue mucosa was an average of $15 \%$ higher in pigs fed CSS vs. LAC and MIX formulas (Additional files 3 and 4). The most abundant class in ileum and colon, Gammaproteobacteria, consisted mostly of a single group unclassified at the genus level but belonging to the family Enterobacteriaceae. The median relative abundance of this group was $66 \%$ across all pigs' ileum and colon contents (Fig. 3). The largest genera in the Clostridia and Bacilli classes were Clostridium sensu stricto and Lactobacillus, respectively. The only other differences we observed across formula diets was an association with higher relative abundance of Clostridium from the clusters XIVa and XI in the colons of piglets receiving LAC-based formula (Fig. 3 and Additional file 5), suggesting these bacteria either benefit from lactose in the diet or are negatively impacted when corn syrup solids are the sole carbohydrate.

Differences between healthy and NEC piglet microbiome We did not observe any difference in the number of observed OTUs between the healthy and NEC groups (Fig. 2). Likewise, at the class level, no differences between healthy and NEC pigs were observed in the mucosal samples (Additional file 6). However, NEC disease was associated with a lower median relative abundance of Gammaproteobacteria in stomach contents and ileum contents, as well as a higher relative abundance of Clostridia in stomach and ileum contents (Additional file 7). The decreased Gammaproteobacteria could be attributed at the family level to lower median relative abundance of Enterobacteriaceae in the stomach contents (6 vs. 19\%) and ileum contents of NEC pigs (51 vs. $73 \%$ in healthy pigs) (Fig. 4). The increased Clostridia could be attributed at the genus level to higher median relative abundance of Clostridium sensu stricto in the ileum contents of NEC pigs (36 vs. $4 \%$ in healthy pigs) (Fig. 4). No major differences at the genus level were observed between healthy and NEC groups in mucosal samples (Additional file 8). 


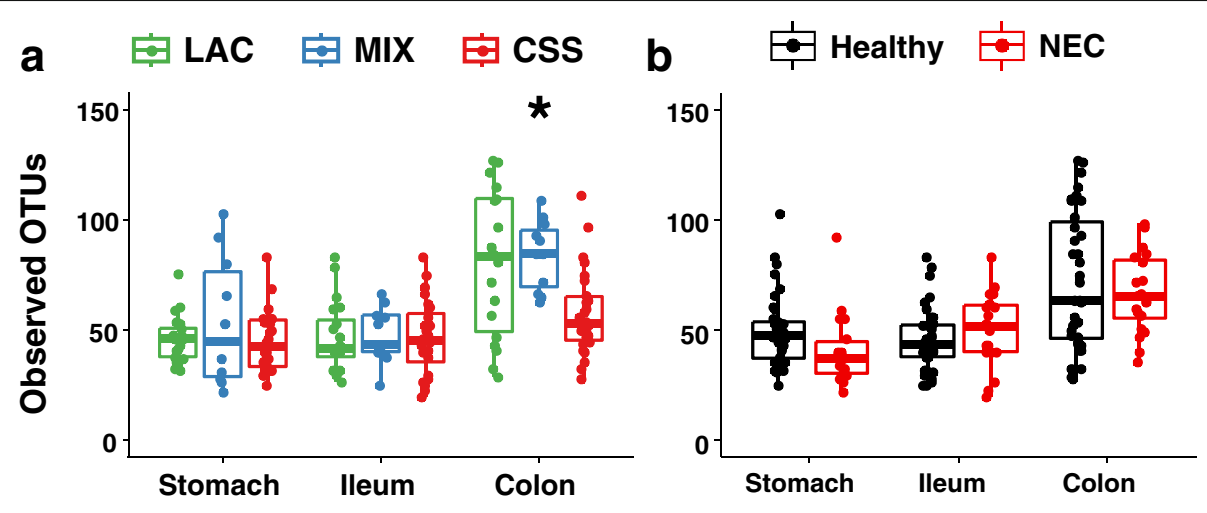

Fig. 2 Microbiota richness in gastrointestinal contents. Counts of observed OTUs for luminal content samples from all pigs comparing the groups at each Gl region in pigs by formula carbohydrate (a) or disease phenotype (b) Holm-Bonferroni adjusted Kruskal-Wallis and Mann-Whitney $U$ tests

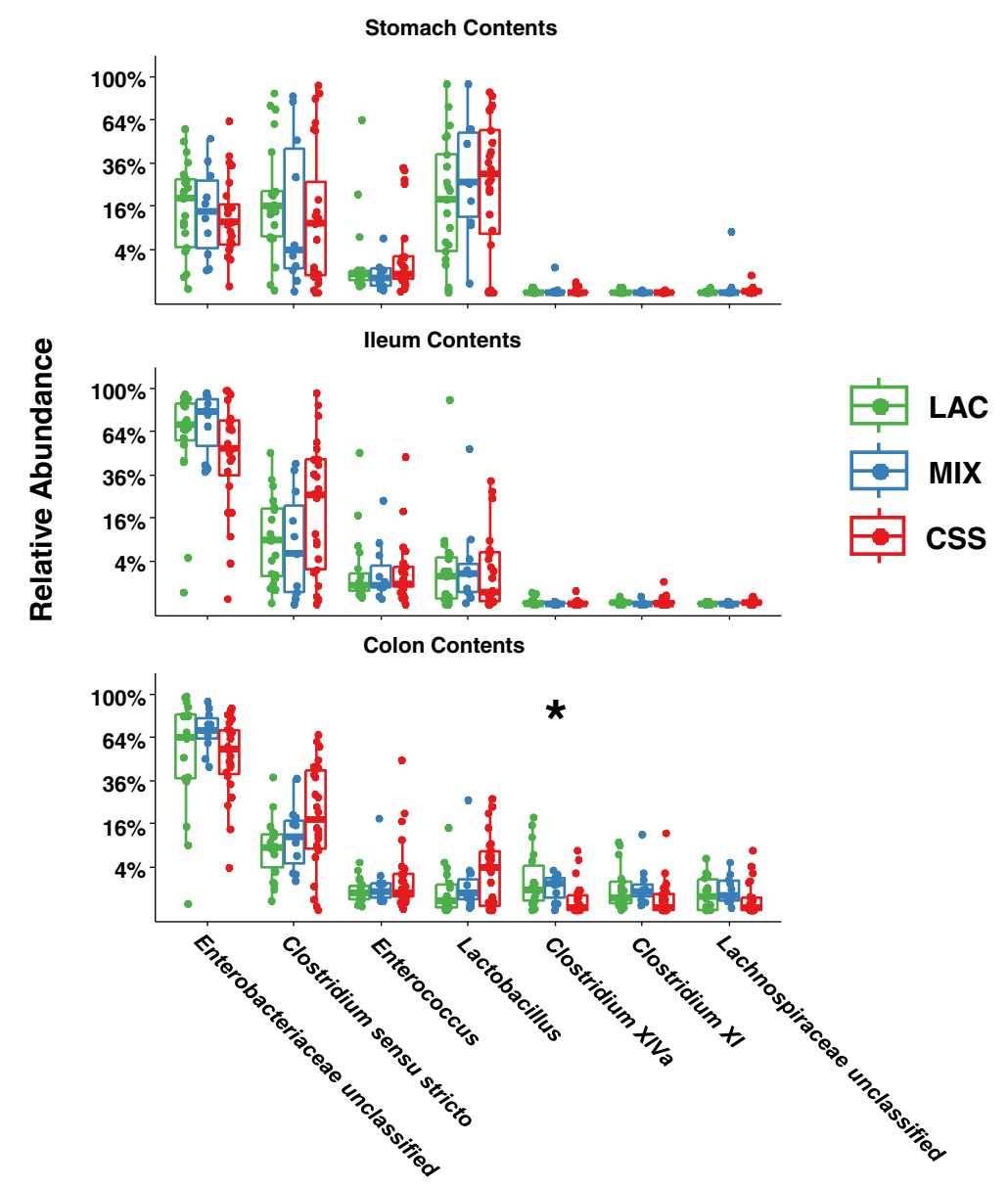

Fig. 3 Relative abundance of the top 7 most abundant genera in gastrointestinal contents, comparing across the different formula groups. Boxand-whisker plots for genus-level comparisons of bacteria detected in piglets' stomach, ileum, and colon luminal contents. Groups include all piglets which were fed one of the three different formulas; Holm-Bonferroni adjusted Kruskal-Wallis tests 


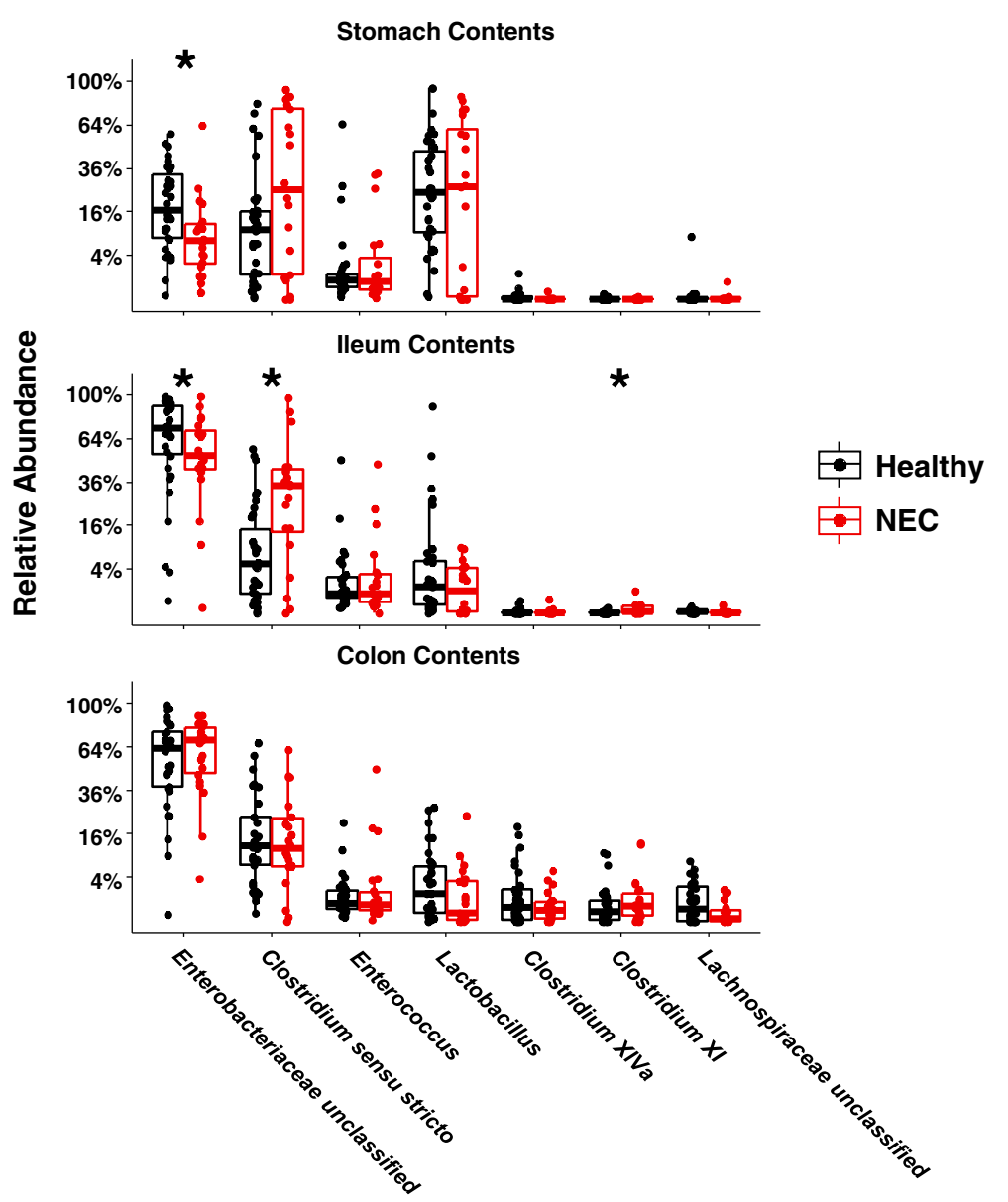

Fig. 4 Relative abundance of the top 7 most abundant genera in gastrointestinal contents, comparing Healthy to NEC. Box-and-whisker plots for genus-level comparisons between piglets which developed NEC and those which did not develop NEC during the course of the experiment, using samples of stomach, ileum, and colon luminal contents from all piglets which were fed one of the three different formulas; Holm-Bonferroni adjusted Mann-Whitney $U$ tests

\section{Metabolomic profiles strongly correlate with formula carbohydrate and NEC disease}

We collected samples of plasma and cecal contents from a subset of the pigs treated in order to assess the metabolic profile as it relates to formula carbohydrates and NEC disease. A total of 425 unique compounds were measured in cecal content samples, and 381 were measured in plasma samples. For both sample types, we performed a two-way analysis of variance with formula carbohydrate and NEC disease phenotype as main effects. Of the 425 measured cecal metabolites, 105 were significantly different across the formula groups, while 52 differed between healthy and NEC. Of the 381 measured plasma metabolites, 4 were significantly different across the formula groups, while 205 differed between healthy and NEC. We did not detect any significant interaction between the two main effects, likely because the group sizes in the study were unequal and not powered sufficiently. Hierarchical clustering and heatmaps of plasma and cecal metabolite profiles helped identify patterns of metabolites that were enriched or depleted in the different groups of interest in this study. Several differences in cecal metabolites were observed between NEC and healthy piglets, including several aromatic amino acid metabolites, polyunsaturated fatty acids, and endocannabinoids (Figs. 5 and 6). Among the plasma metabolites that differed between NEC and healthy piglets were several involved in glycolysis, gluconeogenesis, and pyruvate metabolism pathways (e.g., glucose and lactate), fatty acid metabolism (e.g., acetylcarnitine), hydrolysis of phospholipids (e.g., 2-palmitoylglycerophosphoethanola mine), purine metabolism, aromatic amino acid metabolism, and bile acids (Additional file 9).

\section{Discussion}

The aim of this study was to establish how dietary carbohydrate composition shapes the assembly of the gut microbiome and its metabolic products and whether 


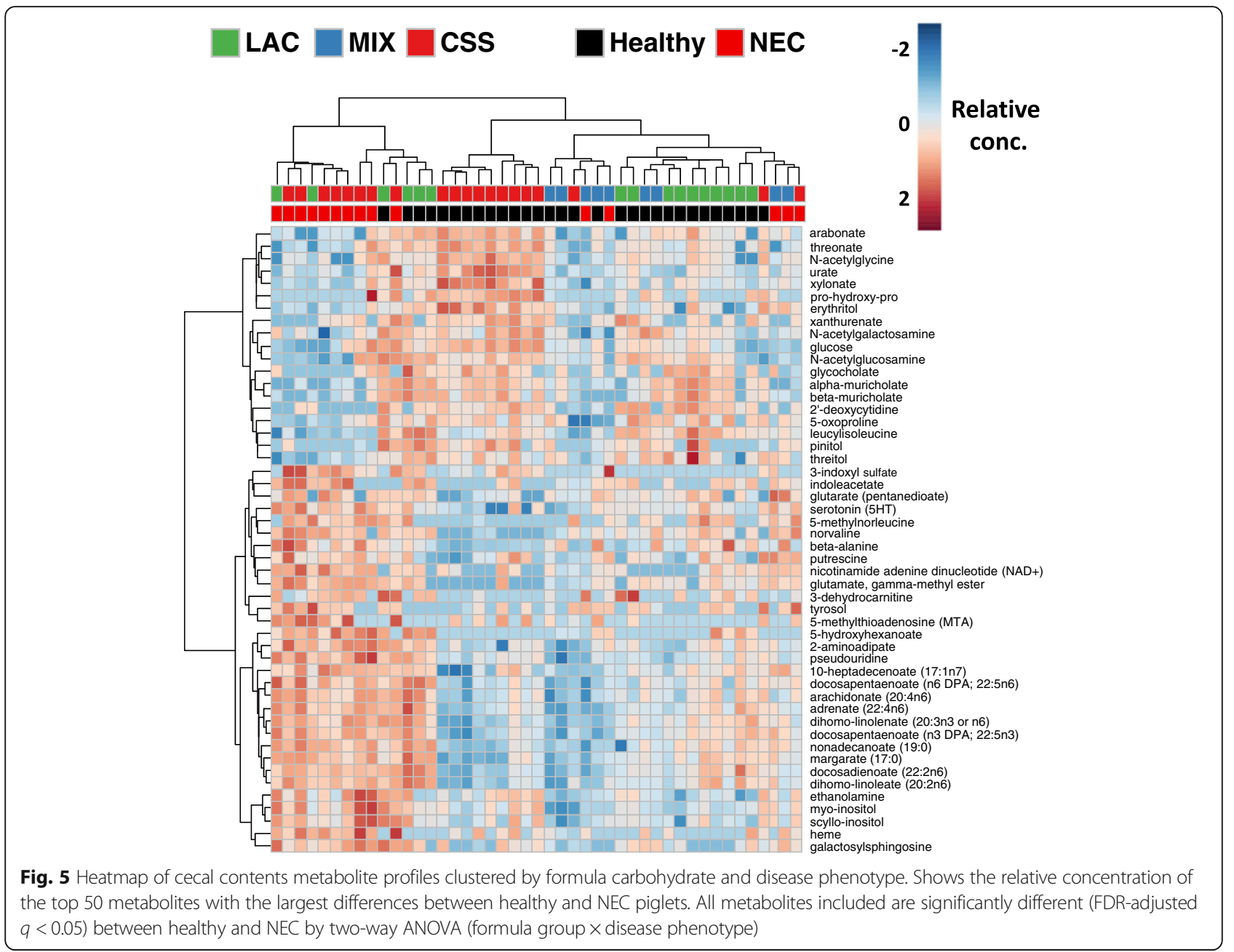

these in turn determine the risk for NEC in premature piglets. Diet has a dominant influence in shaping the gut microbiome of the newborn infant given its role as a source of substrates for bacterial growth. However, the role of specific dietary components on the development of the microbiome in preterm infant is still not fully understood. Although there is great interest in the role of milk oligosaccharides in gut microbiota development, the current study focused on the simpler carbohydrates present in infant formula, lactose, and corn syrup solids. These are the primary carbohydrates in infant formula, yet there is a limited understanding about how they shape the infant gut microbial communities in the context of NEC.

The idea that maldigestion of dietary carbohydrates could lead to NEC goes back to early clinical observations as well as experiments in lactose-intolerant gnotobiotic quail showing that a lactose-based formula led to production of short-chain fatty acids by gut microbes and NEC lesions [7, 56-58]. Our previous studies comparing lactose and glucose polymers (maltodextrin) in neonatal premature piglets, which easily digest lactose but not glucose polymers, also supported the hypothesis that maldigestion can trigger NEC [50]. A few notable changes were made in the current study to increase the clinical relevance, namely continuing the provision of TPN after initiating enteral feeds and gradual increasing the feeding volumes. Importantly, these changes in the clinical protocol lowered the NEC incidence compared to our previous report (as we predicted), but the current study confirms the previous findings of a lower NEC incidence in the piglets fed a lactose- vs. corn syrup solid-based formula.

Despite the evidence that maldigestion of formula dietary carbohydrates can lead to NEC and that gut bacteria seem to mediate this effect, the mechanisms through which this occurs remain unclear. A consistent theme from the many published reports of gut microbiota in NEC is a trend of decreased diversity in NEC infants compared to healthy controls $[18,21,23]$. This is noteworthy considering the gut microbial communities in preterm infants are already very low in diversity compared to older 
children and adults [30, 31, 59, 60]. Likewise, we observed a similar situation in the current study--simple communities with a relatively low number of OTUs and low levels of diversity in all samples. This is also consistent with what has been shown in previous studies on neonatal piglet microbiota [61, 62]. We found no major differences in the inverse Simpson diversity index for Healthy vs. NEC pigs nor across formula groups. This is likely because there are comparatively few OTUs which dominate these young pigs, and diversity metrics are not particularly informative in conditions of just a few dominant species. On the other hand, the observed differences in the number of observed OTUs between CSS-fed piglets and the other diet groups suggest that the newborn piglet microbiota contains a number of low-abundance species which do not grow without lactose in the diet (Fig. 2). Further studies are needed to elucidate the potential significance of the loss of these low-abundance species and how it impacts the developing neonatal gut.

We designed the current study to identify changes in the infant gut microbiome driven by formula carbohydrate and/or NEC disease phenotype. In this study, all the piglets had microbiomes dominated by three classes of bacteria--Gammaproteobacteria, Clostridia, and $\mathrm{Ba}$ cilli, similar to what has been reported in preterm infants [63-65]. Also consistent with studies in preterm human infants delivered by cesarean-section, our preterm piglets had similar low/undetectable levels of $\mathrm{Bac}$ teroidetes and Actinobacteria [32, 66-68]. The use of preterm piglets as a translational model allowed us to characterize distinct microbial communities in specific gastrointestinal regions. One of the most striking differences we observed was the increased relative abundance of Bacilli in stomach samples compared to samples from more distal intestinal regions. Given that we fed formula directly into the stomach, this suggests these are a major group associated with enteral nutrition, consistent with findings in stomach samples from human preterm infants $[69,70]$. This relationship between available substrate and Bacilli may also partly explain why the CSS-fed piglets had higher amounts of Bacilli in their colons, where the maldigested carbohydrate may accumulate. Furthermore, several groups of facultative anaerobic Bacilli, e.g., Lactobacillus, ferment sugars to lactic acid, which may explain the association of cecal lactate with CSS-fed piglets. Overall, the dietary-associated bacterial signatures suggest that the carbohydrate composition of infant formula may affect the microbiota during the early days of development after birth. Relevant to this discussion is evidence in term infants that added dietary lactose can act as a prebiotic to alter the relative abundance of Clostridium, Lactobacillus, and other important genera [71-73]. The role of lactose in shaping the infant gut microbiota and its potential as a prebiotic in the context of low-lactase expressing preterm infants merits further investigation.

A dominant theory tested by multiple clinical studies is that specific groups of gut microbes drive NEC pathogenesis. Several groups of bacteria have been reported to correlate with NEC, yet no consensus pathogens have been confirmed. Microbial signatures associated with NEC include increased Firmicutes [13], for example Clostridia [15, 19, 20, 24], but also decreased Firmicutes [28, 74], such as Clostridia [18, 29], or Negativicutes [29]; increased Gammaproteobacteria [23, 29, 74], especially Enterobacteriaceae [14], for example Klebsiella [15], Enterobacter [22], or Escherichia [16, 20, 75]; decreased Actinobacteria [18], such as Propionibacterium [13] or Bifidobacteria [27]. Indeed, one recent study using a metagenomics approach providing strain-level resolution found that even within an outbreak of NEC at a single NICU and despite harboring similar NEC-associated species, no strains were shared among affected infants [17]. Despite the technical limitations in comparing results across studies, it is obvious that it remains difficult to agree on what might be the core NEC pathogenic taxa. One particularly intriguing idea put forth by some researchers is that although there may be no specific NEC pathogens, perhaps the absence of keystone species during early development may strongly influence the risk of disease. Likely candidates such as Bifidobacteria have been the focus of much research $[27,76]$. But there may be other bacteria which can exert protective influence on the fragile infant. Our observation of higher relative abundance of Clostridium from clusters XIVa and XI in lactose vs. CSS-fed pigs could be another candidate, as species in these genus have been shown to be capable of inducing immune-modulating regulatory T cells [10]. In the current study using preterm piglets, we found significant differences between NEC and healthy in the relative abundance of bacteria within classes which have previously been reported to be associated with NEC such as Clostridia and Gammaproteobacteria. In particular, an NEC-associated increase in ileum Clostridium sensu stricto genus which includes the species Clostridium butyricum and perfringens previously linked to NEC [7, 15, 19, 24]. However, consistent with many reports found in the literature on the NEC microbiome, our results support the conclusion that the presence or absence of any specific taxa does not seem to drive NEC pathogenesis.

In contrast to the large number of studies focused on characterizing the preterm infant's gut microbiome, fewer have used a metabolomic approach to identify NEC-associated metabolites, despite the promise of providing greater mechanistic insight into disease pathogenesis and potential for identifying clinical biomarkers. One of the first such studies looked at urinary metabolite profiles and identified changes in alanine, 
pyridoxine, and histidine [13]. Another analyzed serum of infants with NEC and found a network of metabolites linked to upregulated interleukin-1 $\beta$, including many amino acids [26]. The only published report of metabolomic analysis comparing stool samples from healthy and NEC infants reported five metabolites positively correlated with NEC diagnosis, and these related to the C21-steroid hormone biosynthesis, linoleate metabolism, and leukotriene metabolism and prostaglandin formation from arachidonate pathways [27].

One of the strengths of our study is the combined power of this dual microbiome and metabolomics analysis on paired samples of plasma and cecal contents from the same subjects. Although we found few differences in the gut microbiome profiles associated NEC disease phenotype, there were significant differences in many metabolites between NEC and healthy piglets. Notably, the plasma samples from all NEC pigs, regardless of which formula they received, were enriched with numerous phospholipid metabolites that we suggest are indicative of platelet activation that occurs during disease pathogenesis [77, 78]. Another consistent finding was increased lactate in both plasma of all NEC pigs and cecal contents of CSS-fed pigs. This pattern was intriguing and may signal hypoxic metabolism in the plasma of the host during NEC pathogenesis, whereas increased cecal lactate may be driven by fermentation of corn syrup solids by Lactobacillus as mentioned above. Lactic acidosis, especially the D-isomer produced by bacteria, has been reported in infants with NEC and in conditions of bacterial overgrowth with short-bowel syndrome [79-82]. D-lactic acidosis has been linked to encephalopathy and other toxicities [83]. Among those compounds increased in the cecal contents of NEC piglets, we observed an increase in some products of linoleate, arachidonate, and other omega- 6 fatty acid metabolism, similar to those reported by Stewart et al. The plasma samples from NEC piglets also had increased levels of many of the amino acids reported by Wilcock et al., including ornithine, glycine, serine, and phenylalanine. Among the cecal metabolites in NEC pigs were several metabolites of aromatic amino acids known to be involved in inflammation and metabolized by both the host and microbes, such as serotonin, phenylacetate, and kynurenine [84, 85].

\section{Conclusion}

In summary, we conclude that dietary carbohydrate present in formula can cause specific microbiome changes, yet the association of these changes with disease phenotype was subtle in this study. However, our results suggest that the presence of corn syrup solids, an ingredient that represents over half of the carbohydrate in commercial preterm infant formulas, can lead to major changes in the metabolome that may predispose or even trigger onset of NEC pathology before substantial changes occur in the gut microbiome community. Our results highlight the importance of lactose, the major sugar in human milk, and how it may function as a prebiotic and select for greater microbiome diversity in the developing perinatal gut. More in-depth metagenomic metabolomic analysis is warranted to identify the specific species selected by these dietary carbohydrates and how their fermentation products impact host mucosal function in the developing neonate. Our findings also suggest that the type of carbohydrate in the diet may yield markedly different metabolomic profiles despite relative modest changes in the microbiome community structure.

\section{Methods}

\section{Pig feeding experiment}

The animal protocol was approved by the Animal Care and Use Committee of Baylor College of Medicine and was conducted in accordance with National Institutes of Health guidelines. Mixed-breed pregnant sows were brought to our facility a week before surgery to acclimate. Piglets were delivered by cesarean section at 103 days gestation (full term $=115$ days) as described previously [53]. Following resuscitation and within a few hours after birth, piglets underwent surgery for the placement of a jugular venous catheter and an orogastric tube. Piglets received total parenteral nutrition via jugular catheter for the first $48 \mathrm{~h}$, at which time enteral feeding via orogastric tube was initiated. Passive immunity was provided to the piglets with injections of sow plasma (collected at time of cesarean section) at 6,12 , and $24 \mathrm{~h}$ postnatal. The piglets also received injections (buprenorphine, $0.01 \mathrm{mg} / \mathrm{kg}$ per $12 \mathrm{~h}$ ) for pain and were monitored for blood oxygen saturation level $(\mathrm{SpO} 2)$ and rectal temperature at least twice daily. Over the next 5 days the piglets were fed enterally via orogastric tube every $3 \mathrm{~h}$. The volume of enteral formula fed to the piglets increased each day based on their body weight as measured every other day. As the daily total volume of enteral nutrition increased, the volume of TPN was decreased (see Additional file 10). During the course of the experiment, and especially during the last 5 days when the piglets were receiving enteral formula, signs of NEC were monitored carefully. Initial signs of NEC, e.g., diarrhea and lethargy, were recorded when observed and piglets continued to receive enteral feeds. Upon observation of advanced clinical symptoms of NEC, e.g., abdominal distension, persistent recumbence, and rectal temperature $>40^{\circ} \mathrm{C}$, the piglets were administered subcutaneous buprenorphine $(0.01 \mathrm{mg} / \mathrm{kg}$ every $12 \mathrm{~h}$ while symptoms persist), their $\mathrm{SpO} 2$ was monitored every $3 \mathrm{~h}$, and their enteral feeds continued. Upon observation of these advanced clinical symptoms of NEC and respiratory distress, cyanosis, labored breathing, or persistent 
$\mathrm{SpO} 2<80 \%$, piglets were immediately euthanized with beuthanasia solution. At the end of the study period (7 days total), all remaining piglets were euthanized and their organs were measured and sampled. At the time of sacrifice, a NEC severity score ranging from 1 to 6 was assigned to the major GI regions (stomach, jejunum, ileum, and colon) of each piglet based on the presence of gross pathological signs of NEC disease. A score of 1-2 indicates normal, healthy tissue, 3-4 indicates moderate redness and inflammation, and 5-6 represents pneumatosis and necrosis.

\section{Sample collection and histology}

Samples were collected from the contents of the stomach, distal ileum, and proximal colon (cecum). Tissue mucosal samples were also collected from the mucosa of the ileum and colon. Tissue samples for histology were fixed in $10 \%$ formalin then transferred to $70 \%$ ethanol before being embedded in paraffin, sectioned, and stained with hematoxylin and eosin. For each piglet, sections representing the major GI regions (stomach, jejunum, ileum, and colon) were analyzed under a light microscope by a blinded observer and assigned a histological NEC severity score. The scores ranged from a value of 0 (no damage), or a range of 1 to 4 based on extent of necrosis, villus blunting, and pneumatosis, as described previously [55].

\section{Gene expression}

The expression of several inflammation-related genes was assessed in a subset ( $n=$ LAC-healthy:14, LAC-NEC:2, MIX-healthy:5, MIX-NEC:5, CSS-healthy:9, CSS-NEC:5) of the pigs treated in this study. RNA was extracted from samples of distal ileum using the RNEasy Mini kit (Qiagen). Reverse transcription using the High Capacity cDNA Reverse Transcription (Applied Biosystems) was followed by real-time PCR using Power SYBR Green (ThermoFisher) performed on a Bio-Rad CFX96 machine. The relative expression levels were determined using the delta-delta Ct method.

\section{Microbiome analysis}

For microbiome analysis, we used methods adapted from those used in the NIH Human Microbiome Project [86]. A total of 279 samples from 58 pigs $(n=$ LAC-healthy:18, LAC-NEC:3, MIX-healthy:6, MIX-NEC:5, CSS-healthy:12, CSS-NEC:14) were collected and processed for amplicon sequencing of the V4 region of the 16S ribosomal RNA gene. All samples were processed in two separate batches by the Alkek Center for Metagenomics and Microbiome Research at Baylor College of Medicine. The PowerSoil DNA isolation kit (Mo Bio Laboratories) was used to extract genomic DNA, followed by PCR amplification of the $\mathrm{V} 4$ region of the bacterial $16 \mathrm{~S}$ rRNA gene and sequencing using the $2 \times 250$ paired-end protocol on an Illumina
MiSeq. For technical processes, a set of quality checks are included in all steps from sample intake to data analysis. Projects and samples are inspected as they arrive in the laboratory, and the appropriate paperwork (metadata capture form and chain of custody form) is cross-referenced with the samples received. Samples are logged in and immediately stored in temperature controlled systems until the next step of processing. For extraction, a set of positive and negative controls are included in every processing plate and are used to track extraction efficiencies and cross-contamination. For positive and negative control, there is confirmation of the presence or absence, respectively, of amplicon band at the expected molecular weight after PCR amplification. Positive and negative controls are composed of previously characterized samples and non-template mock samples, respectively. For amplification, a control DNA (bacterial isolate) is included in every amplification plate in addition to a non-template blank. These sets of controls are carried into sequencing and data analysis. Minimum values are required for the following parameters, Q30-Score: $>75 \%$, cluster density: $>700 \mathrm{~K} / \mathrm{mm}^{2}$, clusters pass filter: $>75 \%$, and estimated yield: $>6 \mathrm{~GB}$. Sequence analysis definitions are $>1000$ reads, $99 \%$ of reads mapping to reference, and $<1000$ raw reads, 500 mapped reads for positive and negative controls, respectively. Negative controls were included in one of the two batches and sequence results confirmed the absence of contamination based on findings of 457 raw sequences, 48 mapped sequences, and 17 unmapped sequences.

For our 279 samples, we received back 12,026,340 reads from the sequencer. The raw sequence data was analyzed using the mothur software package (v.1.39.0) [87]. Sequence reads having 0 ambiguous bases, max length of 260 , and max homopolymer length of 8 were retained. Reads were aligned to the V4 region of the SILVA database (v.123). Chimeras were removed using vsearch using the default parameters and singleton reads were also removed. Reads were then classified using the RDP training set (v.14), and OTUs were formed by clustering at the 0.03 level using opticlust [88]. Subsequent analysis using the mothur-outputted biom file was performed in R using, among others, the phyloseq package [89]. A total of 273 samples passed quality filtering and read processing. The average number of sequences per sample was 28,010 , and the standard deviation was 9880 . After calculating bacterial community richness (observed OTU counts) and alpha diversity (inverse Simpson's index), the data were subsampled to the minimum sequence count by sample type, e.g., contents $(11,790)$ or mucosal tissue $(5315)$, and the OTU abundances were transformed to relative proportions by dividing each by the total counts per sample. All OTUs assigned to a given taxonomic ranking, e.g., class or genus, were combined together and subsequent comparisons were made using these taxonomic groupings. 


\section{Metabolomic analysis}

Metabolomic analysis of plasma and cecal contents were analyzed in collaboration with Metabolon, Inc. (Durham, NC). A total of 90 samples, 45 of each sample type, from 45 pigs $\quad(n=$ LAC-healthy:14, LAC-NEC:2, MIX-healthy:5, MIX-NEC:4, CSS-healthy:11, CSS-NEC:9) were collected and processed for non-targeted metabolomics profiling (Additional file 11). Briefly, small molecules were extracted from the samples using a combination of aqueous and organic solvents. Residual organic solvent was removed using a TurboVap (Zymark), and the extracts were lyophilized, then equal amounts were analyzed by GC/MS and UPLC-MS/MS in parallel. For UPLC-MS/MS, extracts were analyzed under both acidic and basic conditions using an ACQUITY (Waters) UPLC and an LTQ (Thermo-Finnigan) mass spectrometer. For GC/MS, extracts were derivatized with bistrimethyl-silyl-triflouroacetamide, then analyzed using a Trace DSQ (Thermo-Finnigan) mass spectrometer.
Compounds were identified by comparison of the raw data with Metabolon's curated library of standards. The values for compounds in the cecal contents samples were normalized by the dry mass of the sample. Missing values were imputed with half the compound minimum. Absolute compound intensity values were used to calculate fold differences between the groups, while for all other analyses, the values were transformed using the generalized log transformation then mean-centered and scaled by the standard deviation.

\section{Statistical analysis}

All results were analyzed using the $\mathrm{R}$ software program for statistics and graphical presentation [90]. The following statistical techniques were used to determine significance of a given observation: For NEC incidence Fisher's exact test was used. For the survival curve analysis, a log-rank test was used. For severity measurements, a linear model with
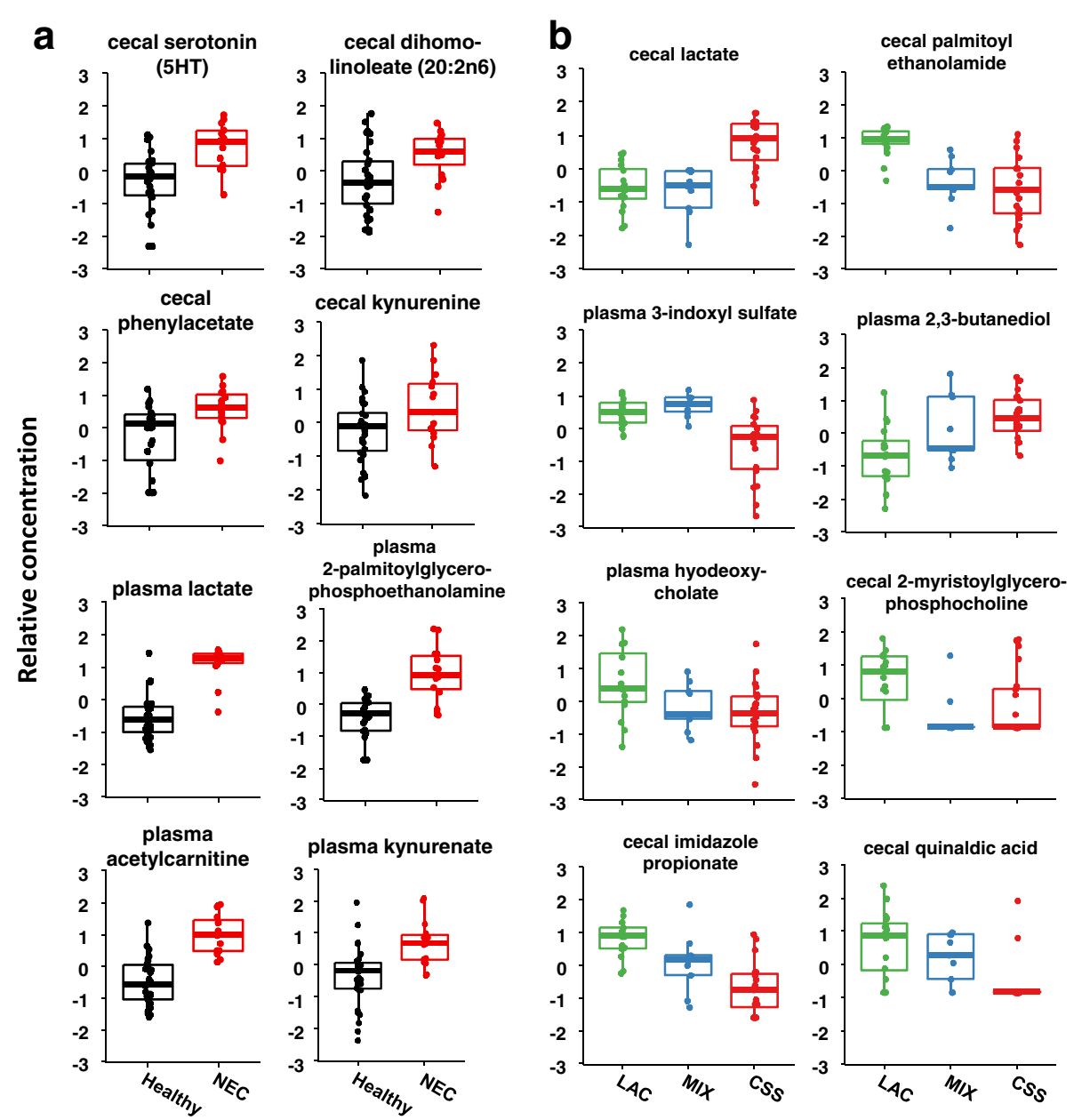

Fig. 6 Selected plasma and cecal metabolites with differences by disease phenotype (a) or formula carbohydrate (b). Representative metabolites from the major pathways found to be differentially abundant in plasma and cecal content samples. Metabolite concentrations are shown on a mean-centered and standard deviation-scaled $y$-axis. Each metabolite shown was found to be significantly different (FDR-adjusted $q<0.01$ ) either between healthy and NEC piglets or across formula groups by two-way ANOVA (formula group $\times$ disease phenotype) 
birthweight and farm as covariates and Tukey's post hoc comparisons was used. For relative gene expression, pairwise Mann-Whitney $U$ tests were used. In analyzing relative abundance of bacterial classes and genera, a Kruskal-Wallis rank sum test was used to test for differences across the three formula groups, a Mann-Whitney $U$ test was used to test for differences between healthy and $\mathrm{NEC}$, and to control for significance tests involving multiple hypotheses, the Holm-Bonferroni correction was applied and significance was assessed at alpha $<0.05$. For the analysis of the metabolomics profiles, a two-way analysis of variance (ANOVA) was used with formula group (LAC, MIX, and CSS) and disease phenotype (NEC and Healthy) as main effects. We initially performed analysis to test for an interaction and when the interaction was not significant main effects were reported. The Benjamini-Hochberg false discovery rate method, with $q<0.01$, was used to prevent inflation of the type I error rate.

\section{Additional files}

Additional file 1: Plot of terminal ileum villus height in individual pigs from newborn, healthy, and NEC groups over the entire study period. (PDF $80 \mathrm{~kb}$ )

Additional file 2: Gene expression of major inflammatory cytokines. Samples from distal ileum tissue from a subset of the pigs in this study were assessed for expression of inflammation-related genes using real-time reverse-transcription PCR (RT-PCR). Holm-Bonferroni adjusted Mann-Whitney U test. (PDF 64 kb)

Additional file 3: Relative abundance of the top 4 most abundant classes in intestinal mucosal tissue, comparing across the different formula groups. Box-and-whisker plots for class-level comparisons of bacteria detected in piglets' ileum and colon mucosal tissue. Groups include all piglets which were fed one of the three different formulas; Holm-Bonferroni adjusted Kruskal-Wallis tests. (PDF $140 \mathrm{~kb}$ )

Additional file 4: Relative abundance of the top 4 most abundant classes in gastrointestinal contents, comparing across the different formula groups. Box-and-whisker plots for class-level comparisons of bacteria detected in piglets' stomach, ileum, and colon luminal contents. Groups include all piglets which were fed one of the three different formulas; Holm-Bonferroni adjusted Kruskal-Wallis tests. (PDF 162 kb)

Additional file 5: Relative abundance of the seven most abundant genera in intestinal mucosal tissue, comparing across the different formula groups. Box-and-whisker plots for genus-level comparisons of bacteria detected in piglets' ileum and colon mucosal tissue. Groups include all piglets which were fed one of the three different formulas; Holm-Bonferroni adjusted Kruskal-Wallis tests. (PDF $185 \mathrm{~kb}$ )

Additional file 6: Relative abundance of the four most abundant classes in intestinal mucosal tissue, comparing Healthy to NEC. Box-and-whisker plots for class-level comparisons between piglets which developed NEC and those which did not develop NEC during the course of the experiment, using samples of ileum and colon mucosal tissue from all piglets which were fed one of the three different formulas; Holm-Bonferroni adjusted Mann-Whitney U tests. (PDF $138 \mathrm{~kb}$ )

Additional file 7: Relative abundance of the four most abundant classes in gastrointestinal contents, comparing Healthy to NEC. Box-and-whisker plots for class-level comparisons between piglets which developed NEC and those which did not develop NEC during the course of the experiment, using samples of stomach, ileum, and colon luminal contents from all piglets which were fed one of the three different formulas; Holm-Bonferroni adjusted Mann-Whitney $U$ tests. (PDF $159 \mathrm{~kb}$ )
Additional file 8: Relative abundance of the seven most abundant genera in intestinal mucosal tissue, comparing Healthy to NEC. Box-andwhisker plots for genus-level comparisons between piglets which developed NEC and those which did not develop NEC during the course 41 of the experiment, using samples of ileum and colon mucosal tissue from all piglets which were fed one of the three different formulas; Holm-Bonferroni adjusted Mann-Whitney U tests. (PDF $183 \mathrm{~kb}$ )

Additional file 9: Heatmap of plasma metabolite profiles clustered by formula carbohydrate and disease phenotype. Shows the relative concentration of the top 50 metabolites with the largest differences between healthy and NEC piglets. All metabolites included are significantly different (FDR-adjusted $q<0.05$ ) between healthy and NEC by two-way ANOVA (formula group $\times$ disease phenotype). (PDF $117 \mathrm{~kb}$ )

Additional file 10: Overview of the study design and experimental groups. Diagram of the timing of major components of the experiment, including introduction and weaning of total parenteral nutrition (TPN), introduction and ramp-up of enteral formula feeding, and the time of euthanasia and tissue collection for the three experimental groups. (PDF 86 kb)

Additional file 11: Description of metabolomic platform, analysis pipeline, and compound identification. (DOCX $16 \mathrm{~kb}$ )

Additional file 12: Data, metadata files, and R scripts used to generate the figures. The Rmarkdown_data_metadata.zip is a compressed folder containing the Rmarkdown file, which details the analysis scripts (in both RMD and HTML format). The batch file used to process the sequence data in mothur is provided. Microbiome data is included here as a BIOMformatted table. All other data (metabolomics and cytokine expression) along with metadata are CSV files. (ZIP $992 \mathrm{~kb}$ )

\section{Abbreviations}

CSS: Corn syrup solids; LAC: Lactose; NEC: Necrotizing enterocolitis;

OTU: Operational taxonomic unit

\section{Acknowledgements}

We are grateful to Liwei Cui, Jessica Donnelly, and Irving Zamora for their assistance with this study. We thank Rob Shulman and Robert Britton for review of this manuscript prior to publication.

\section{Funding}

This work was supported in part by federal funds from the USDA, Agricultural Research Service under Cooperative Agreement Number 309251000-060-01, and grants Mead Johnson Nutrition, the Alkek Center for Metagenomics and Microbiome Research (CMMR), and the Texas Medical Center Digestive Diseases Center (NIH Grant P30 DK-56338). Lee Call was supported by training fellowships from the National Institutes of Health Grant T32-GM088129 and Gulf Coast Consortia, NLM Training Program in Biomedical Informatics (T15-LM007093).

\section{Availability of data and materials}

The microbiome sequence data supporting the conclusions of this article have been deposited in the NCBI Sequence Read Archive with links to BioProject accession number PRJNA397167 in the NCBI BioProject database (https://www.ncbi.nlm.nih.gov/bioproject/). All other data files along with the $\mathrm{R}$ scripts used to generate the main figures for this paper are made available in a zipped folder as Additional file 12.

\section{Authors' contributions}

$\mathrm{LC}, \mathrm{BS}, \mathrm{BO}, \mathrm{FS}, \mathrm{OO}$, and $\mathrm{DB}$ performed the experiments and analyzed the data. LC, NA, and JP contributed to the sequencing data analysis and interpretation. $L C$ and DB wrote and edited the manuscript. BB, AW, and RW contributed to the study design, data interpretation, and scientific discussions. All authors read and approved the final manuscript.

\section{Ethics approval}

The animal protocol was approved by the Animal Care and Use Committee of Baylor College of Medicine and was conducted in accordance with National Institutes of Health guidelines.

\section{Consent for publication}

Not applicable 


\section{Competing interests}

D.G.B. received grant support for this work from Mead Johnson Nutrition and the Alkek Center for Metagenomic and Microbiome Research. B.B., A.W., and R.W. were employees of Mead Johnson Nutrition during the completion of this research. Other authors declare that they have no competing interests.

\section{Publisher's Note}

Springer Nature remains neutral with regard to jurisdictional claims in published maps and institutional affiliations.

\section{Author details}

'USDA-ARS Children's Nutrition Research Center, Department Pediatric Gastroenterology, Hepatology, and Nutrition, Baylor College of Medicine, 1100 Bates Ave, Houston, TX 77030, USA. ${ }^{2}$ Division of Pediatric Surgery, Baylor College of Medicine, 6701 Fannin St, Suite 1210, Houston, TX 77030, USA. ${ }^{3}$ Alkek Center for Metagenomics and Microbiome Research, Baylor College of Medicine, One Baylor Plaza, MS BCM385, Houston, TX 77030, USA. ${ }^{4}$ Mead Johnson Pediatric Nutrition Institute, 2400 W Lloyd Expressway, Evansville, IN 47712, USA.

Received: 20 March 2018 Accepted: 8 June 2018

Published online: 19 June 2018

\section{References}

1. Lin PW, Stoll BJ. Necrotising enterocolitis. Lancet. 2006;368:1271-83. https:// doi.org/10.1016/S0140-6736(06)69525-1.

2. Neu J, Walker WA. Necrotizing enterocolitis. N Engl J Med. 2011;364:255-64.

3. Aagaard K, Ma J, Antony KM, Ganu R, Petrosino J, Versalovic J. The placenta harbors a unique microbiome. Sci Transl Med 2014;6:237ra265. doi:https:// doi.org/10.1126/scitranslmed.3008599.

4. Ardissone AN, de la Cruz DM, Davis-Richardson AG, Rechcigl KT, Li N, Drew $\mathrm{JC}$, et al. Meconium microbiome analysis identifies bacteria correlated with premature birth. PLoS One. 2014;9:e90784. https://doi.org/10.1371/journal. pone.0090784

5. Musemeche CA, Kosloske AM, Bartow SA, Umland ET. Comparative efects of ischemia, bacteria, and substrate on the pathogenesis of intestinal necrosis. J Pediatr Surg. 1986;21:536-8.

6. Sangild PT, Siggers RH, Schmidt M, Elnif J, Bjornvad CR, Thymann T, et al. Diet- and colonization-dependent intestinal dysfunction predisposes to necrotizing enterocolitis in preterm pigs. Gastroenterology. 2006;130:177692. https://doi.org/10.1053/j.gastro.2006.02.026.

7. Waligora-Dupriet A-J, Dugay A, Auzeil N, Huerre M, Butel M-J. Evidence for clostridial implication in necrotizing enterocolitis through bacterial fermentation in a gnotobiotic quail model. Pediatr Res. 2005;58:629-35. https://doi.org/10.1203/01.PDR.0000180538.13142.84.

8. Jensen ML, Thymann T, Cilieborg MS, Lykke M, Mølbak L, Jensen BB, et al. Antibiotics modulate intestinal immunity and prevent necrotizing enterocolitis in preterm neonatal piglets. Am J Physiol Gastrointest Liver Physiol. 2014;306:G59-71. https://doi.org/10.1152/ajpgi.00213.2013.

9. Round JL, Mazmanian SK. Inducible Foxp3+ regulatory T-cell development by\na commensal bacterium of the intestinal microbiota. Proc Natl Acad Sci U S A. 2010;107:12204-9.

10. Atarashi K, Tanoue T, Shima T, Imaoka A, Kuwahara T, Momose Y, et al. Induction of colonic regulatory $T$ cells by indigenous Clostridium species. Science. 2011;331:337-41. https://doi.org/10.1126/science.1198469.

11. Ivanov II, Atarashi K, Manel N, Brodie EL, Shima T, Karaoz U, et al. Induction of intestinal Th17 cells by segmented filamentous bacteria. Cell. 2009;139: 485-98. https://doi.org/10.1016/j.cell.2009.09.033.

12. Smith B, Bodé S, Petersen BL, Jensen TK, Pipper C, Kloppenborg J, et al. Community analysis of bacteria colonizing intestinal tissue of neonates with necrotizing enterocolitis. BMC Microbiol. 2011;11:73. https://doi.org/10.1186/ 1471-2180-11-73.

13. Morrow AL, Lagomarcino AJ, Schibler KR, Taft DH, Yu Z, Wang B, et al. Early microbial and metabolomic signatures predict later onset of necrotizing enterocolitis in preterm infants. Microbiome. 2013;1:13. https://doi.org/10. 1186/2049-2618-1-13.

14. Claud EC, Keegan KP, Brulc JM, Lu L, Bartels D, Glass E, et al. Bacterial community structure and functional contributions to emergence of health or necrotizing enterocolitis in preterm infants. Microbiome. 2013;1:20. https://doi.org/10.1186/2049-2618-1-20.
15. Sim K, Shaw AG, Randell P, Cox MJ, Mcclure ZE, Li M, et al. Dysbiosis anticipating necrotizing enterocolitis in very premature infants. 2015;60:389-97.

16. Ward DV, Scholz M, Zolfo M, Taft DH, Schibler KR, Tett A, et al. Metagenomic sequencing with strain-level resolution implicates uropathogenic E. coli in necrotizing enterocolitis and mortality in preterm infants. Cell Rep. 2016;14: 2912-24. https://doi.org/10.1016/j.celrep.2016.03.015.

17. Raveh-Sadka T, Thomas BC, Singh A, Firek B, Brooks B, Castelle CJ, et al. Gut bacteria are rarely shared by co-hospitalized premature infants, regardless of necrotizing enterocolitis development. Elife. 2015;2015:1-25.

18. McMurtry VE, Gupta RW, Tran L, Blanchard EE, Penn D, Taylor CM, et al. Bacterial diversity and clostridia abundance decrease with increasing severity of necrotizing enterocolitis. Microbiome. 2015;3:11. https://doi.org/ 10.1186/s40168-015-0075-8.

19. Cassir N, Benamar S, Khalil JB, Croce O, Saint-Faust M, Jacquot A, et al. Clostridium butyricum strains and dysbiosis linked to necrotizing enterocolitis in preterm neonates. Clin Infect Dis. 2015;61:1107-15.

20. Zhou Y, Shan G, Sodergren E, Weinstock G, Walker WA, Gregory KE. Longitudinal analysis of the premature infant intestinal microbiome prior to necrotizing enterocolitis: a case-control study. PLoS One. 2015;10:1-16.

21. Brower-Sinning R, Zhong D, Good M, Firek B, Baker R, Sodhi CP, et al. Mucosaassociated bacterial diversity in necrotizing enterocolitis. PLoS One. 2014;9

22. Stewart CJ, Marrs ECL, Magorrian S, Nelson A, Lanyon C, Perry JD, et al. The preterm gut microbiota: changes associated with necrotizing enterocolitis and infection. Acta Paediatr Int J Paediatr. 2012;101:1121-7.

23. Wang Y, Hoenig JD, Malin KJ, Qamar S, Petrof EO, Sun J, et al. 16 S rRNA gene-based analysis of fecal microbiota from preterm infants with and without necrotizing enterocolitis. ISME J. 2009;3:944-54. https://doi.org/10. 1038/ismej.2009.37.

24. Rozé JC, Ancel PY, Lepage P, Martin-Marchand L, Al NZ, Delannoy J, et al. Nutritional strategies and gut microbiota composition as risk factors for necrotizing enterocolitis in very-preterm infants. Am J Clin Nutr. 2017;106:821-30.

25. Stewart CJ, Nelson A, Treumann A, Skeath T, Cummings SP, Embleton ND, et al. Metabolomic and proteomic analysis of serum from preterm infants with necrotising entercolitis and late onset sepsis. Pediatr Res. 2016;79:42531. https://doi.org/10.1038/pr.2015.235.

26. Wilcock A, Begley P, Stevens A, Whatmore A, Victor S. The metabolomics of necrotising enterocolitis in preterm babies: an exploratory study. J Matern Fetal Neonatal Med. 2016;29:758-62. https://doi.org/10.3109/14767058.2015.1017462

27. Stewart CJ, Embleton ND, Marrs ECL, Smith DP, Nelson A, Abdulkadir B, et al. Temporal bacterial and metabolic development of the preterm gut reveals specific signatures in health and disease. Microbiome. 2016:1-10. https://doi.org/10.1186/s40168-016-0216-8.

28. Pammi M, Cope J, Tarr PI, Warner BB, Morrow AL, Mai V, et al. Intestinal dysbiosis in preterm infants preceding necrotizing enterocolitis: a systematic review and meta-analysis. Microbiome. 2017;5:1-15.

29. Warner BB, Deych E, Zhou Y, Hall-Moore C, Weinstock GM, Sodergren E, et al. Gut bacteria dysbiosis and necrotising enterocolitis in very low birthweight infants: a prospective case-control study. Lancet. 2016;387: 1928-36. https://doi.org/10.1016/S0140-6736(16)00081-7.

30. Koenig JE, Spor A, Scalfone N, Fricker AD, Stombaugh J, Knight R, et al. Succession of microbial consortia in the developing infant gut microbiome. Proc Natl Acad Sci U S A 2011;108 Suppl:4578-4585.

31. Yatsunenko T, Rey FE, Manary MJ, Trehan I, Dominguez-Bello MG, Contreras $M$, et al. Human gut microbiome viewed across age and geography. Nature. 2012;486:222-7. https://doi.org/10.1038/nature11053.

32. Bäckhed F, Roswall J, Peng Y, Feng Q, Jia H, Kovatcheva-Datchary P, et al. Dynamics and stabilization of the human gut microbiome during the first year of life. Cell Host Microbe. 2015;17:690-703.

33. Hiergeist A, Gläsner J, Reischl U, Gessner A. Analyses of intestinal microbiota: culture versus sequencing. ILAR J. 2015;56:228-40.

34. Clooney AG, Fouhy F, Sleator RD, O'Driscoll A, Stanton C, Cotter PD, et al. Comparing apples and oranges?: next generation sequencing and its impact on microbiome analysis. PLoS One. 2016;11:1-16.

35. Van den Abbeele P, Van de Wiele T, Verstraete W, Possemiers S. The host selects mucosal and luminal associations of coevolved gut microorganisms: a novel concept. FEMS Microbiol Rev. 2011;35:681-704.

36. Kostic AD, Howitt MR, Garrett WS. Exploring host--microbiota interactions in animal models and humans. Genes Dev. 2013;27:701-18.

37. Chung H, Pamp SJ, J a H, Surana NK, Edelman SM, Troy EB, et al. Gut immune maturation depends on colonization with a host-specific microbiota. Cell. 2012;149:1578-93. https://doi.org/10.1016/j.cell.2012.04.037. 
38. Nguyen TL, Vieira-Silva S, Liston ARJ. How informative is the mouse for human gut microbiota research? Dis Model Mech. 2015;8:1-16.

39. Lucas a, Cole TJ. Breast milk and neonatal necrotising enterocolitis. Lancet. 1990;336:1519-23. https://doi.org/10.1016/0140-6736(90)93304-8.

40. Meinzen-Derr J, Poindexter B, Wrage L, Morrow AL, Stoll B, Donovan EF. Role of human milk in extremely low birth weight infants' risk of necrotizing enterocolitis or death. J Perinatol. 2009;29:57-62. https://doi.org/10.1038/jp.2008.117.

41. Cristofalo EA, Schanler RJ, Blanco CL, Sullivan S, Trawoeger R, KiechlKohlendorfer $\mathrm{U}$, et al. Randomized trial of exclusive human milk versus preterm formula diets in extremely premature infants. J Pediatr. 2013;163: 1592-1595.e1. https://doi.org/10.1016/j.jpeds.2013.07.011.

42. Sullivan S, Schanler RJ, Kim JH, Patel AL, Traw??ger R, Kiechl-Kohlendorfer U, et al. An exclusively human milk-based diet is associated with a lower rate of necrotizing enterocolitis than a diet of human milk and bovine milkbased products. J Pediatr. 2010;156

43. Schanler RJ, Shulman RJ, Lau C. Feeding strategies for premature infants: beneficial outcomes of feeding. Pediatrics. 1999;103:1150-7.

44. Shulman RJ, Schanler RJ, Lau C, Heitkemper M, Ou C-N, Smith EO. Early feeding, feeding tolerance, and lactase activity in preterm infants. J Pediatr. 1998;133:645-9. https://doi.org/10.1016/S0022-3476(98)70105-2.

45. Griffin MP, Hansen JW. Can the elimination of lactose from formula improve feeding tolerance in premature infants? J Pediatr. 1999;135:587-92.

46. Kien CL. Lactose in formulas for preterm infants. J Pediatr. 2001;138:148-9. https://doi.org/10.1067/mpd.2001.108205.

47. Shulman RJ, Wong WW, Smith EOB. Influence of changes in lactase activity and small-intestinal mucosal growth on lactose digestion and absorption in preterm infants. Am J Clin Nutr. 2005;81:472-9.

48. Bode L. Human milk oligosaccharides: every baby needs a sugar mama. Glycobiology. 2012;22:1147-62. https://doi.org/10.1093/glycob/cws074.

49. Autran CA, Kellman BP, Kim JH, Asztalos E, Blood AB, Spence ECH, et al. Human milk oligosaccharide composition predicts risk of necrotising enterocolitis in preterm infants. Gut. 2017;:.gutjnl-2016-312819; https://doi. org/10.1136/gutjnl-2016-312819.

50. Thymann T, Møller HK, Stoll B, Støy ACF, Buddington RK, Bering SB, et al. Carbohydrate maldigestion induces necrotizing enterocolitis in preterm pigs. Am J Physiol Gastrointest Liver Physiol. 2009;297:G1115-25. https://doi. org/10.1152/ajpgi.00261.2009.

51. Sangild PT, Thymann T, Schmidt M, Stoll B, Burrin DG, Buddington RK. Invited review: the preterm pig as a model in pediatric gastroenterology. J Anim Sci. 2013;91:4713-29. https://doi.org/10.2527/jas.2013-6359.

52. Oosterloo BC, Premkumar M, Stoll B, Olutoye O, Thymann T, Sangild PT, et al. Dual purpose use of preterm piglets as a model of pediatric Gl disease. Vet Immunol Immunopathol. 2014;159:156-65. https://doi.org/10.1016/j. vetimm.2014.02.012

53. Ghoneim N, Bauchart-Thevret C, Oosterloo B, Stoll B, Kulkarni M, de Pipaon MS, et al. Delayed initiation but not gradual advancement of enteral formula feeding reduces the incidence of necrotizing enterocolitis (NEC) in preterm pigs. PLoS One. 2014;9:e106888. https://doi.org/10.1371/journal. pone.0106888

54. Zamora IJ, Stoll B, Ethun CG, Sheikh F, Yu L, Burrin DG, et al. Low abdominal NIRS values and elevated plasma intestinal fatty acid-binding protein in a premature piglet model of necrotizing enterocolitis. PLoS One. 2015;10: e0125437. https://doi.org/10.1371/journal.pone.0125437.

55. Benight NM, Stoll B, Olutoye OO, Holst JJ, Burrin DG. GLP-2 delays but does not prevent the onset of necrotizing enterocolitis in preterm pigs. J Pediatr Gastroenterol Nutr. 2013;56:623-30. https://doi.org/10.1097/MPG. Ob013e318286891e.

56. Book LS, Herbst JJ, Jung L. Carbohydrate malabsorption in necrotizing enterocolitis. J Pediatr. 1976;57:201-4

57. Kien CL. Colonic fermentation of carbohydrate in the premature infant: possible relevance to necrotizing enterocolitis. J Pediatr. 1990;117:S52-8.

58. Butel MJ, Roland N, Hibert a, Popot F, Favre a, Tessedre a C, et al. Clostridial pathogenicity in experimental necrotising enterocolitis in gnotobiotic quails and protective role of bifidobacteria. J Med Microbiol. 1998;47:391-9.

59. Moles L, Gómez M, Heilig H, Bustos G, Fuentes S, de Vos W, et al. Bacterial diversity in meconium of preterm neonates and evolution of their fecal microbiota during the first month of life. PLoS One. 2013;8:e66986. https:// doi.org/10.1371/journal.pone.0066986.

60. Hopkins MJ, Sharp R, Macfarlane GT, Bank S. Age and disease related changes in intestinal bacterial populations assessed by cell culture, $16 \mathrm{~S}$ rRNA abundance, and community cellular fatty acid profiles Age and disease related changes in intestinal bacterial populations assessed by cell culture. November 2007;1(2001):198-205.

61. Tannock GW, Fuller R, Pedersen K. Lactobacillus succession in the piglet digestive tract demonstrated by plasmid profiling. Appl Environ Microbiol. 1990;56:1310-6.

62. Frese SA, Parker K, Calvert CC, Mills DA. Diet shapes the gut microbiome of pigs during nursing and weaning. Microbiome. 2015;3:28. https://doi.org/10. 1186/s40168-015-0091-8.

63. La Rosa PS, Warner BB, Zhou Y, Weinstock GM, Sodergren E, Hall-Moore CM, et al. Patterned progression of bacterial populations in the premature infant gut. Proc Natl Acad Sci. 2014;111:1409497111 https://doi.org/10.1073/pnas. 1409497111.

64. Costello EK, Carlisle EM, Bik EM, Morowitz MJ, Relman DA. Microbiome assembly across multiple body sites in low-birthweight infants. MBio. 2013;4:1-12.

65. Arboleya S, Binetti A, Salazar N, Fernández N, Solís G, Hernández-Barranco A, et al. Establishment and development of intestinal microbiota in preterm neonates. FEMS Microbiol Ecol. 2012;79:763-72.

66. Barrett E, Kerr C, Murphy K, O'Sullivan O, Ryan CA, Dempsey EM, et al. The individual-specific and diverse nature of the preterm infant microbiota. Arch Dis Child Fetal Neonatal Ed. 2013;98:F334-40. https://doi.org/10.1136/ archdischild-2012-303035.

67. Jakobsson HE, Abrahamsson TR, Jenmalm MC, Harris K, Quince C, Jernberg $\mathrm{C}$, et al. Decreased gut microbiota diversity, delayed Bacteroidetes colonisation and reduced Th1 responses in infants delivered by caesarean section. Gut. 2014;63:559-66. https://doi.org/10.1136/gutjnl-2012-303249.

68. Bokulich NA, Chung J, Battaglia T, Henderson N, Jay M, Li H, et al. Antibiotics, birth mode, and diet shape microbiome maturation during early life. Sci Transl Med. 2016;8:343ra82. https://doi.org/10.1126/scitranslmed.aad7121.

69. Moles L, Gómez M, Jiménez E, Bustos G, de Andrés J, Melgar A, et al. Bacterial diversity of the gastric content of preterm infants during their first month of life at the hospital. Front Nutr 2017;4 April. doi:https://doi.org/10. 3389/fnut.2017.00012

70. Patel K, Konduru K, Patra AK, Chandel DS, Panigrahi P. Trends and determinants of gastric bacterial colonization of preterm neonates in a NICU setting. PLoS One. 2015;10:1-21.

71. Szilagyi A. Redefining lactose as a conditional prebiotic. Can J Gastroenterol. 2004;18:163-7.

72. Szilagyi A, Shrier I, Heilpern D, Je JS, Park S, Chong G, et al. Differential impact of lactose/lactase phenotype on colonic microflora. Can J Gastroenterol. 2010:24:373-9.

73. Francavilla R, Calasso M, Calace L, Siragusa S, Ndagijimana M, Vernocchi $P$, et al. Effect of lactose on gut microbiota and metabolome of infants with cow's milk allergy. Pediatr Allergy Immunol. 2012;23:420-7.

74. Mai V, Young CM, Ukhanova M, Wang X, Sun Y, Casella G, et al. Fecal microbiota in premature infants prior to necrotizing enterocolitis. PLoS One. 2011;6:e20647. https://doi.org/10.1371/journal.pone.0020647.

75. Stewart CJ, Marrs ECL, Nelson A, Lanyon C, Perry JD, Embleton ND, et al. Development of the preterm gut microbiome in twins at risk of necrotising enterocolitis and sepsis. PLoS One. 2013;8:e73465. https://doi.org/10.1371/ journal.pone.0073465.

76. Underwood MA, German JB, Lebrilla CB, Mills DA. Bifidobacterium longum subspecies infantis: champion colonizer of the infant gut. Pediatr Res. 2015;77:229-35.

77. Caplan MS, Hsueh W. Necrotizing enterocolitis: role of platelet activating factor, endotoxin, and tumor necrosis factor. J Pediatr. 1990;117:0-4.

78. Duvernay MT, Matafonov A, Lindsley CW, Hamm HE. Platelet lipidomic profiling: novel insight into cytosolic phospholipase A2a activity and its role in human platelet activation. Biochemistry. 2015;54:5578-88.

79. Lei G, Zhang J, Wang X, Chen M. Plasma D-lactate levels in necrotizing enterocolitis in premature infants. Iran J Pediatr. 2016;26:10-2.

80. Mayeur C, Gratadoux JJ, Bridonneau C, Chegdani F, Larroque B, Kapel N, et al. Faecal $D / L$ lactate ratio is a metabolic signature of microbiota imbalance in patients with short bowel syndrome. PLoS One. 2013;8:1-12.

81. Uchida H, Yamamoto H, Kisaki Y, Fujino J, Ishimaru Y, Ikeda H. D-lactic acidosis in short-bowel syndrome managed with antibiotics and probiotics. J Pediatr Surg. 2004;39:634-6.

82. Bongaerts GP, Tolboom JJ, Naber AH, Sperl WJ. Role of bacteria in the pathogenesis of short bowel syndrome-associated D-lactic acidemia. Microb Pathog. 1997;22:285-93.

83. Petersen C. D-Lactic Acidosis. Nutr Clin Pract 2005:20 December:634-645. 
84. Ciorba MA. Indoleamine 2,3 dioxygenase in intestinal disease. Curr Opin Gastroenterol. 2013;29:146-52. https://doi.org/10.1097/MOG. 0b013e32835c9cb3.

85. Margolis KG, Gershon MD. Enteric neuronal regulation of intestinal inflammation. Trends Neurosci. 2016;39:614-24.

86. Aagaard K, Petrosino J, Keitel W, Watson M, Katancik J, Garcia N, et al. The human microbiome project strategy for comprehensive sampling of the human microbiome and why it matters. FASEB J. 2012;27:1012-22. https:// doi.org/10.1096/fj.12-220806.

87. Schloss PD, Westcott SL, Ryabin T, Hall JR, Hartmann M, Hollister EB, et al. Introducing mothur: open-source, platform-independent, communitysupported software for describing and comparing microbial communities. Appl Environ Microbiol. 2009;75:7537-41.

88. Westcott SL, Schloss PD. OptiClust, an improved method for assigning amplicon-based sequence data to operational taxonomic units. mSphere. 2017;2:e00073-17. https://doi.org/10.1128/mSphereDirect.00073-17.

89. McMurdie PJ, Holmes S. phyloseq: an R package for reproducible interactive analysis and graphics of microbiome census data. PLoS One. 2013;8:e61217. https://doi.org/10.1371/journal.pone.0061217.

90. R Core Team. R: a language and environment for statistical computing. In: R foundation for statistical computing; 2016. https://www.r-project.org/.

Ready to submit your research? Choose BMC and benefit from:

- fast, convenient online submission

- thorough peer review by experienced researchers in your field

- rapid publication on acceptance

- support for research data, including large and complex data types

- gold Open Access which fosters wider collaboration and increased citations

- maximum visibility for your research: over $100 \mathrm{M}$ website views per year

At BMC, research is always in progress.

Learn more biomedcentral.com/submissions 